\title{
Sur la voie des Achats Responsables : le cas de la SNCF*
}

\author{
On the way to PSR: the SNCF Case \\ Rachel Bocquet \\ rachel.bocquet@univ-smb.fr \\ Elodie Gardet \\ elodie.gardet@univ-smb.fr \\ Lauriane Robert \\ lauriane.robert@univ-smb.fr
}

Classification JEL : M14, D22

Résumé :

L’objectif de cette recherche est de mieux comprendre le processus d'adoption d'une démarche d'Achats Responsables (AR). Peu de travaux se sont intéressés à ce processus ainsi qu’à ses antécédents intra et inter-organisationnels. L’étude empirique, basée sur le cas exemplaire de la SNCF, révèle que la démarche d'AR suit un processus en trois phases (mise en place, déploiement, maintien) et que les deux types d’antécédents jouent un rôle différencié selon ces phases. Ils montrent aussi le rôle clé joué par certains acteurs déjà convaincus pour accroître l'adhésion à la démarche. Cette recherche débouche sur des recommandations utiles aux entreprises désireuses de s’engager dans une démarche d’AR ou soucieuses de la pérenniser.

Mots-clés : Achats Responsables - Antécédents intra et inter-organisationnels - Processus

* Les auteurs remercient tout particulièrement la SNCF ainsi que THESAME et l'ensemble des partenaires du programme PEAK (Purchasing European Alliance for Knowledge) destiné à développer les relations collaboratives clients-fournisseurs. 


\section{Abstract}

The purpose of this study is to gain a better understanding of Purchasing Social Responsibility (PSR) adoption process. Few studies exist on PSR adoption process and its intra and interorganizational antecedents. Based on the exemplary case of the SNCF, results support that PSR adoption process can be split into three stages (setting-up, operating, sustaining). They also show that antecedents differ according to those stages. Finally, results also shed some light on the key role of actors, who personally embrace CSR values and get others involved in this process. This research provides useful implications for firms which would engage into a PSR process or maintain such a process.

Keywords: Purchasing Social Responsibility - Intra and inter-organizational antecedents - Process. 


\section{Introduction}

Les stratégies d'achats coercitives (Leire et Mont, 2010) qui consistent à imposer unilatéralement aux fournisseurs des réductions de coûts et de délais ont montré leurs limites. De récents scandales, tels que celui de la viande de cheval en France, témoignent des conséquences néfastes associées à ce type de stratégie ${ }^{1}$. Dans ce contexte, les Achats Responsables (AR) deviennent une préoccupation centrale des entreprises. Ce constat est d'ailleurs mis en évidence dans une étude récente de l’ObsAR (Observatoire des Achats Responsables, 2013) qui indique que $75 \%$ des grandes entreprises privées ont adopté une politique d'AR ${ }^{2}$. Toutefois, force est de constater qu'elles ne sont pas toutes à la même phase d'avancement. Si 75 \% diffusent en interne leur démarche d’AR en communiquant sur la démarche menée en interne, seulement la moitié d'entre elles suivent les actions menées par la mise en place d'indicateurs de performance.

Cette hétérogénéité dans les pratiques ne trouve pas encore d'explication convaincante au plan théorique pour deux raisons essentielles. Premièrement, les recherches sur l'adoption d'une démarche d'AR restent encore peu développées et lorsqu'elles existent, celles-ci considèrent l'adoption essentiellement comme une décision prise à un instant $t$ (Carter et Jennings, 2002 ; Blome et Paulraj, 2013). Ces recherches, qui proposent une vision statique, ont toutefois l'avantage d'identifier deux grandes catégories d'antécédents explicatifs de la décision d'adopter : les antécédents intra et / ou inter-organisationnels. Deuxièmement, les rares travaux qui proposent une vision processuelle de la démarche (Zsidisin et Siferd, 2001 ; Maignan et al., 2002 ; Theodorakopoulos et al., 2005 ; Andersen et Skjoett-Larsen, 2009 ; Leire et Mont, 2010) ne permettent pas de comprendre le passage d'une phase à l'autre. En combinant ces deux approches, cette recherche vise à comprendre la mise en œuvre d'une démarche d'AR. Nous défendons donc ici la thèse selon laquelle les antécédents ne sont pas statiques et qu’ils évoluent au fur et à mesure de l’avancée de la démarche d’AR. Notre méthodologie, de type abductif, aboutit ainsi à des propositions d'explications plutôt qu'à des règles indiscutables. Une telle perspective peut permettre, au moins en partie, de mettre en lumière l'origine

\footnotetext{
${ }^{1}$ Cet exemple montre que « c'est la recherche du prix toujours plus bas qui éclaire le mieux toute cette affaire et qui justifie aussi tous les débordements, voire les escroqueries » (les Échos du 25/02/2013). Ainsi, le primat accordé à la seule baisse des coûts a conduit un fournisseur à acheter de la viande de cheval $30 \%$ moins cher en sacrifiant toute traçabilité et responsabilité vis-à-vis des consommateurs.

2 Résultats basés sur la 4ème enquête « baromètre des Achats Responsables » de l’ObsAR, établis sur un échantillon de 238 entreprises dont la part des plus grandes (>1000 salariés) représente $46 \%$.
} 
les différences observées quant à la phase d'avancement des entreprises en matière d'AR et d’identifier les conditions favorables à la pérennité de cette démarche.

L'article est structuré comme suit : la partie 1 fournit une définition de l'objet d'étude, les $\mathrm{AR}$, en tant que décision puis comme processus. Une revue systématique de la littérature des principaux antécédents intra et inter-organisationnels est réalisée afin de comprendre leur influence selon les différentes phases du processus. La partie 2 justifie le choix de la SNCF pour son caractère exemplaire et non pour son statut d'entreprise publique et présente la méthodologie mise en œuvre. Dans la troisième partie, les résultats sont présentés puis discutés. La conclusion aborde les apports et les implications managériales de cette recherche, ses limites et les perspectives de recherches futures.

\section{Le processus d'Achats Responsables et ses antécédents}

Après avoir précisé ce que recouvrent concrètement les Achats Responsables (AR), il s’agit de les définir dans une approche statique puis dynamique. Une analyse des principaux antécédents identifiés par la littérature est ensuite proposée. L’enjeu de cette recherche est de comprendre si ces antécédents varient en fonction des différentes phases du processus d’AR.

\subsection{Les achats Responsables : de la décision au processus}

D'après la norme Achats Responsables de l'AFNOR (norme NFX 50-135 et future ISO 20400), les AR reposent sur l'existence d'un compromis entre la dimension environnementale, sociale et économique dans l'intérêt de l'ensemble des parties prenantes. Ils se distinguent donc des achats « classiques » qui restent centrés sur la recherche d'une performance économique du donneur d'ordre à court terme autour du triptyque qualité, coût, délai (Walker et Brammer, 2009).

Cette norme permet de définir le périmètre des AR autour de 7 questions centrales qui concernent 1) la gouvernance de l'organisation, 2) les droits de l'homme, 3) les relations et conditions de travail, 4) l'environnement, 5) les pratiques des affaires, 6) les questions relatives aux consommateurs, 7) l'engagement sociétal. Ces questions donnent aux entreprises un cadre pour décliner des pratiques concrètes à mettre en œuvre en matière d'AR. À titre d'exemple, pour veiller au respect de l'environnement, une entreprise impliquée dans des AR s'engagera à n'acheter aucune substance toxique dangereuse pour l'homme et l'environnement. Un autre exemple peut être fourni sur la thématique des relations et des conditions de travail. Dans ce cadre, l'entreprise pourra privilégier l'appel à des personnes n'ayant pas un accès facilité à 
l'emploi pour cause de handicap ou de manque de qualification. D’ailleurs, certaines familles d'achats sont aujourd'hui raisonnées par le recours à des sous-traitants œuvrant dans le domaine social, comme les EA (Entreprise Adaptée) ou encore les ESAT (Établissement et Service d'Aide par le Travail).

Au plan théorique, le concept d'AR a fait l'objet d'une littérature croissante. Dès les années 1990, dans un contexte où l'environnement devient une réelle préoccupation pour la société, Drumwright (1994) insiste sur l'importance de considérer des critères non économiques dans la gestion des achats. Carter et Jennings (2002) sont à l'origine du concept de PSR (Purchasing Social Responsibility) et de ses principaux composants : droits de l'homme, diversité, environnement, sécurité, communauté et philanthropie. Ce concept de PSR a été mobilisé dans de nombreuses recherches, avec des terminologies quelque peu différentes, telles que Socially Responsible Purchasing (Leire et Mont, 2010) ou encore Sustainable Procurement (Walker et al., 2008 ; Walker et Brammer, 2009). Nous utiliserons dans cette recherche le terme d'AR et retiendrons, dans une vision statique, la définition de Drumwright (1994, p.1) : «[Les AR] prennent en compte les conséquences publiques des achats des organisations ou suscitent des changements sociaux positifs au travers des pratiques achats non économiques c'est-à-dire des critères autres que le prix ou les compromis entre le prix et les diverses dimensions de la qualité ». Le terme d'AR est, à ce jour, le plus communément accepté dans la littérature francophone, repris d'ailleurs par l'AFNOR et par l’ObsAR.

En dépit d'une littérature croissante, les auteurs ayant introduit une vision processuelle au sein de leur définition des AR sont encore rares. Theodorakopoulos et al. (2005) proposent une adaptation du modèle de Supply Chain Learning (Bessant et al., 2003) appliqué aux AR en intégrant la question de la diversité des fournisseurs. Ils identifient un processus en trois phases : « une phase initiale de mise en place », « une phase de déploiement opérationnel » et « une phase de maintien/entretien». Malgré l’identification de trois phases, les auteurs se concentrent sur la première (i.e. la décision d'adopter) et n’étudient pas les deux autres. Nous avons identifié également d'autres modèles dynamiques des AR fondés sur 4, 5 ou 6 étapes (Maignan et al., 2002 ; Zsidisin et Siferd, 2001 ; Andersen et Skjoett-Larsen, 2009 ; Leire et Mont, 2010). Pour construire la grille d'analyse, nous retenons le processus en trois phases de Theodorakopoulos et al. (2005). À la différence des autres modèles, il ne s’agit pas de décrire la mise en place de codes de conduite du point de vue de la firme focale (Andersen et SkjoettLarsen, 2009) vis-à-vis de fournisseurs traités comme une «black box » (Emmelhainz et Adams, 1999) ou de simples destinataires (Maignan et al., 2002). De plus, les trois séquences 
de mise en place des AR dépassent une vision strictement interne du processus en intégrant la question de l'intégration des fournisseurs à cette démarche, à travers notamment le développement de leurs capacités d'apprentissage. Le processus de mise en œuvre des AR étant loin d'être automatique, la phase de maintien est pleinement intégrée alors qu'elle est sousestimée, voire absente des autres modèles (e.g. Emmelhainz et Adams, 1999). Si le découpage en trois phases proposé par Theodorakopoulos et al. (2005) présente également l'avantage d’être facilement opérationnalisable, sa pertinence sera questionnée lors de l'étude empirique. À cette étape, il devient possible d'affiner la définition donnée des AR vus comme processus en nous référant aux phases préalablement identifiées. Les AR peuvent être appréhendés comme le résultat d'un processus à trois phases (mise en place, déploiement, maintien) qui prend en compte les conséquences publiques des achats par l’intégration, outre des critères économiques, des critères sociaux et environnementaux.

2.2. Les antécédents intra et inter-organisationnels nécessaires à la mise en œuvre d'une démarche $\mathrm{AR}$

Les achats sont l'interface de l'entreprise vis-à-vis de ses partenaires économiques, notamment ses fournisseurs, sans lesquels aucune activité d'entreprise ne peut exister (Calvi, 2000 ; Blome et Paulraj, 2013). Ils font également le lien avec toutes les activités internes en traduisant une réalité économique et de marché vers l’interne (Carter et Jennings, 2002). Ce rôle d'interface entre l'interne et l'externe, affirmé dans le modèle de Theodorakopoulos et al. (2005), justifie l'importance d'analyser les antécédents intra et inter-organisationnels pour comprendre le processus d'adoption d'une démarche d'AR.

\subsubsection{Les antécédents intra-organisationnels}

Les travaux sur les AR permettent de mettre en lumière plusieurs antécédents internes favorables à la mise en œuvre de la démarche. L’engagement sincère, qu’il soit basé sur des valeurs (Drumwright, 1994 ; Carter et Carter, 1998) ou qu'il relève de la morale (Drumwright 1994) est un antécédent majeur. L’implication de la direction en faveur d'une politique d'AR joue également un rôle essentiel (Drumwright, 1994 ; Carter et Carter, 1998 ; Emmelhainz et Adams, 1999 ; Carter et Jennings, 2000 ; Zsidisin et Siferd, 2001 ; Walker et Brammer, 2009 ; Hoejmose et Adrien-Kirby, 2012). En raison de leur position hiérarchique et leur rôle stratégique, les dirigeants ont le pouvoir de prendre des décisions et de définir des politiques et programmes intégrant la Responsabilité Sociale de l'Entreprise (Blome et Paulraj, 2013). La direction par son leadership et sa propre conduite a aussi la possibilité d’influencer 
le comportement de ses employés (Carter et Jennings, 2000). Outre le rôle du top management, l’implication du middle management apparaît également comme un antécédent clé (Carter et Carter, 1998 ; Zsidisin et Siferd, 2001). Carter et Carter (1998) montrent notamment que ce dernier peut a minima faciliter une adoption progressive des pratiques d'achats environnementales. Au-delà de l'implication du management, le niveau de formalisation joue un rôle crucial dans la mise en œuvre effective de la démarche. La définition et la diffusion de règles explicites telles qu'une politique, un programme, des codes d'éthique ou de conduite, ou encore des certifications sont des antécédents importants à condition qu'ils ne soient pas que des «mesures de façade » (Carter et Jennings, 2002 ; Worthington et al., 2008) et qu'ils soient suffisamment ambitieux (Igarashi et al., 2013). Par exemple, les codes d'éthique informent les employés sur les comportements non acceptables ; les politiques d'entreprise et, à un niveau plus infra, les politiques de services facilitent la définition d'objectifs (Carter et Jennings, 2000). Ces règles formelles permettent de structurer et de communiquer sur la démarche et ainsi d'améliorer la coordination (Carter et Jennings, 2000). La mise en œuvre de la démarche est également facilitée par la formation et la montée en expertise en interne (Leire et Mont, 2010).

Le tableau 1 synthétise cette revue de la littérature des principaux antécédents intraorganisationnels à l'œuvre dans le cadre d'une démarche d'AR. Afin de pouvoir les regrouper de manière cohérente, des travaux récents montrent que les approches organisationnelles offrent un cadre adapté alors qu'elles sont très peu mobilisées (Sarkis et al., 2011 ; Hoejmose et Adrien-Kirby, 2012) ${ }^{3}$. Dans cette perspective, notre recherche s'appuie sur l'approche de Burns et Stalker (1961) qui, bien qu'ancienne, permet de distinguer les organisations plutôt organiques des organisations davantage mécanistes en fonction de leur niveau de centralisation, de spécialisation et de formalisation. Cette approche propose une vision relativement intégrée de l'organisation adaptée à notre questionnement. D’abord, elle permet de différentier deux types distincts d’organisations (organisations mécanistes versus organiques) à partir de dimensions bien identifiées et opérationnalisables (Cooper et Zmud, 1990 ; Hult et al., 2000 ;

\footnotetext{
${ }^{3}$ La lecture institutionnaliste et des parties prenantes ont largement été mobilisées dans les travaux portant sur les antécédents. Cependant, ces théories permettent d'expliquer plutôt les leviers externes. Tate et $a l$. (2012), Sarkis et al. (2011) et Hoejmose et Kirby (2012) montrent que les AR sont un champ récent qui offre la possibilité d'être exploré au travers de théories organisationnelles qui ont peu ou pas été mobilisées à ce jour. Ces auteurs préconisent d'utiliser d'autres approches que l'approche traditionnelle des parties prenantes. Leur constat est que les approches organisationnelles sont peu mobilisées alors qu'elles offrent un cadre adapté pour expliquer les facteurs explicatifs des AR.
} 
Sine et al., 2006). En même temps, elle échappe à une conception dualiste en proposant un continuum plus apte à saisir la variété et la complexité des situations : les organisations ne rentrant pas parfaitement dans une catégorie ou une autre, même si des tendances se dégagent (Burns et Stalker, 1961). Enfin, cette approche permet de structurer le foisonnement des antécédents intra-organisationnels issus de la littérature et de les regrouper de manière cohérente en proposant une grille de lecture adaptée aux AR.

\section{Tableau 1 : Antécédents intra-organisationnels et type de structure}

\begin{tabular}{|c|c|c|c|}
\hline \multirow{2}{*}{$\begin{array}{l}\text { Antécédents et leurs } \\
\text { caractéristiques }\end{array}$} & \multicolumn{2}{|c|}{ Type de structure } & \multirow[b]{2}{*}{ Principaux auteurs } \\
\hline & Mécaniste & Organique & \\
\hline Formalisation & Élevée $(++)$ & $\longrightarrow$ Faible (-) & \\
\hline Langage & écrit & oral & $\begin{array}{l}\text { Andersen et Skjoett- } \\
\text { Larsen (2009), Blome et } \\
\text { Paulraj (2013) }\end{array}$ \\
\hline Standardisation & $\begin{array}{c}\text { élevée (règles, standards, } \\
\text { procédures) }\end{array}$ & $\begin{array}{l}\text { faible (ajustements mu- } \\
\text { tuels, face à face) }\end{array}$ & Carter et Carter (1998) \\
\hline Règles & explicites & implicites & $\begin{array}{l}\text { Carter et Jennings (2000, } \\
\text { 2002), Worthington et } a l \text {. } \\
\text { (2008) }\end{array}$ \\
\hline Incitation & extrinsèque & intrinsèque & $\begin{array}{l}\text { Andersen et Skjoett- } \\
\text { Larson (2009) ; Closs et } \\
\text { al. (2011), Hoejmose et } \\
\text { Adrien-kirby (2012) }\end{array}$ \\
\hline $\begin{array}{l}\text { Indicateurs de perfor- } \\
\text { mance }\end{array}$ & quantitatifs & qualitatifs & $\begin{array}{l}\text { Drumwright (1994) ; Car- } \\
\text { ter et Jennings (2000) }\end{array}$ \\
\hline Centralisation & Élevée $(++)$ & $\rightarrow$ Faible (-) & \\
\hline Coordination & faite par la hiérarchie & $\begin{array}{l}\text { faite par toute } \\
\text { l'organisation }\end{array}$ & Carter et Jennings (2004) \\
\hline Communication & $\begin{array}{l}\text { verticale (ascendante et } \\
\text { descendante) }\end{array}$ & verticale et latérale & Closs et al. (2011) \\
\hline Management & directif & participatif & Drumwright (1994) \\
\hline Spécialisation & Élevée (++) & $\rightarrow$ Faible (-) & \\
\hline Responsabilité & limitée aux experts & diffuse & Drumwright (1994) \\
\hline Savoir & concentré & disséminé & $\begin{array}{l}\text { Andersen et Skjoett- } \\
\text { Larson (2009) ; Leire et } \\
\text { Mont (2010) }\end{array}$ \\
\hline $\begin{array}{l}\text { Interactions entre ser- } \\
\text { vices }\end{array}$ & rares & fréquentes & Drumwright (1994) \\
\hline
\end{tabular}


La grande majorité des travaux présentés lors de cette revue de littérature n’intègrent pas une analyse processuelle et certaines contradictions apparaissent. Par exemple, Drumwright (1994) montre que l'adoption d'une culture informelle a un impact positif sur le déploiement de la démarche alors que Carter et Jennings (2002) ou Worthington et al. (2008) prônent la définition et la diffusion de règles explicites. Ces résultats vont dans le sens de la thèse que nous défendons ici, à savoir que les antécédents intra-organisationnels ne sont pas statiques et qu'ils peuvent évoluer au fur et à mesure de l'avancée de la démarche. Ainsi, les antécédents seraient différents selon les trois phases repérées, d’où la possibilité de réconcilier certains résultats a priori contradictoires. Cette thèse permettrait également de comprendre comment, certaines entreprises engagées dans un même processus d'adoption d'AR, parviennent mieux que d'autres à franchir les différentes phases. Ainsi, le problème n'est plus tant de lister les leviers intra-organisationnels d'une telle démarche, mais bien de les identifier pour chacune des phases dans la mesure où ils peuvent être distincts.

Outre les antécédents intra-organisationnels, les achats sont également caractérisés par leur relation avec l'extérieur et notamment avec les fournisseurs.

\subsubsection{Les antécédents inter-organisationnels}

La concurrence s'exerce désormais au niveau de la supply chain et la performance de l'entreprise dépend aussi de son réseau de partenaires et notamment de ses fournisseurs (Cousins et Spekman, 2003). Les relations clients-fournisseurs sont traditionnellement examinées dans la littérature sur les antécédents des AR selon deux types d'approches, économiques (avec comme cadre théorique les théories contractuelles, en particulier la théorie des coûts de transaction, pour analyser les relations inter-organisationnelles) versus sociologiques (la sociologie, grâce à la vision dynamique des relations a mis l'accent sur les processus d’interaction pour comprendre les relations sociales (Burt, 1982 ; Reynaud, 1988)).

De ces deux grandes approches émergent deux principaux antécédents pour caractériser les relations inter-organisationnelles dans le cadre d'une démarche d'AR. Il s'agit des antécédents relationnels et des antécédents contractuels ( $c f$. Tableau 2). Ils sont tantôt substituables tantôt complémentaires. Lorsqu'ils sont considérés comme substituables, les relations clientsfournisseurs sont assimilées soit à des contrats et des incitations, soit à de la confiance, de la réputation et du partage de valeurs communes (Poppo et Zenger, 2002). Lorsqu'ils sont complémentaires, les clients et leurs fournisseurs sont placés dans un cadre relationnel, considéré comme un complément au contrat. Ainsi, les dimensions transactionnelles s'avèrent nécessaires, mais non suffisantes pour expliquer à elles seules les relations d'échange. La prise en 
compte explicite des dimensions sociales et temporelles devient essentielle (Reynaud, 1988). Dans ce cadre, les antécédents formels (notamment les contrats) et informels (notamment la confiance) ne sont plus appréhendés de manière antinomique. Les antécédents relationnels peuvent garantir l'exécution des contrats formels grâce à l'élaboration d'étroites relations de confiance et à une meilleure compréhension mutuelle des membres de la coopération (Poppo et Zenger, 2002).

Dans les relations clients-fournisseurs, les antécédents hérités des approches économiques englobent notamment les mécanismes formels tels que les contrats, les procédures et les cahiers des charges (Andersen et Skjoett-Larsen, 2009 ; Gimenez et Sierra, 2013). Les contrôles et les visites fournisseurs, complétés par des systèmes de mesure de leur performance, apparaissent comme des antécédents favorables à la performance des fournisseurs (Gimenez et Sierra, 2013). Outre la signature d'un contrat, Carter et Jennings (2000) recommandent de développer des modes de communication formels et d'élaborer des règles explicites telles que les codes d'éthique ou la mise en place de certifications (ex. : ISO 14 000). Ils considèrent que la coordination structurée et formelle des acteurs joue favorablement sur le déploiement d'une démarche d'AR. Enfin, des antécédents liés à des mécanismes d'incitation/sanction représentent également des leviers importants pour mettre en œuvre une démarche d'AR. Une évaluation formelle permet de mesurer la performance des fournisseurs, de les comparer, et de leur apporter des suggestions d'améliorations. Au regard de l'approche sociale, la mise en œuvre d'un programme d'AR nécessite des dispositifs de communication et de l'investissement en ressources pour développer les capacités d'apprentissage du fournisseur (Theodorakopoulos et al., 2005). Gimenez et Sierra (2013) montrent que le passage d'une stratégie réactive à une stratégie proactive en matière de mise en œuvre d'un programme de Résponsabilité Sociale d'Entreprise (RSE) se caractérise par une augmentation du degré d'évaluation et de collaboration avec le fournisseur. Carter et Jennings (2000) rappellent que la communication est un antécédent à la confiance. Or, la confiance et l'historique des relations apparaissent également comme des antécédents importants à la mise en œuvre d'une démarche d'AR. En effet, l'ancienneté de la relation client-fournisseur constitue un préalable pour la mise en place d'exigences environnementales et sociales. La capacité à créer des relations étroites à long terme avec les fournisseurs et partenaires stratégiques est un élément déterminant (Theodorakopoulos et al., 2005). Afin de développer ces relations long terme, le niveau d'implication du client chez le fournisseur apparaît également comme un antécédent important. Le fait que le client s'implique directement chez le fournisseur afin de former, 
d'apporter une expertise (Krause et al., 1999) ou de suivre les actions correctives (Andersen et Skjoett-Larsen, 2009) joue un rôle direct et décisif dans la qualité de la relation.

Le tableau 2 ci-après propose une synthèse des principaux travaux sur les antécédents interorganisationnels et les classifie selon le type d'approche retenue des relations interorganisationnelles.

Tableau 2 - Antécédents inter-organisationnels et type d'approche

\begin{tabular}{|c|c|c|c|}
\hline \multirow[b]{2}{*}{$\begin{array}{c}\text { Antécédents et leurs carac- } \\
\text { téristiques }\end{array}$} & \multicolumn{2}{|c|}{ Approche } & \multirow[b]{2}{*}{ Principaux auteurs } \\
\hline & Économique & Sociale & \\
\hline Contractuel & Élevée (++) & $\longrightarrow$ Faible (-) & \\
\hline Régulation de l'échange & $\begin{array}{l}\text { forte contractualisa- } \\
\text { tion }\end{array}$ & $\begin{array}{c}\text { Faible contractua- } \\
\text { lisation }\end{array}$ & $\begin{array}{l}\text { Leire et Mont (2010) ; } \\
\text { Hoejmose et Adrien-Kirby } \\
\text { (2012) ; Gimenez et Sierra } \\
\text { (2013) }\end{array}$ \\
\hline Place de la concurrence & élevée & faible & $\begin{array}{l}\text { Carter et Carter (1998) ; } \\
\text { Park-Poaps et Rees (2010) }\end{array}$ \\
\hline $\begin{array}{l}\text { Système d'incitations et de } \\
\text { sanctions }\end{array}$ & explicite & implicite & $\begin{array}{l}\text { Maignan et al. (2002) ; } \\
\text { Andersen et Skjoett-Larsen } \\
\text { (2009) }\end{array}$ \\
\hline Contrôle formel & élevé & faible & $\begin{array}{c}\text { Vachon et Klassen (2008) ; } \\
\text { Andersen et Skjoett-Larsen } \\
\text { (2009) ; Leire et Mont } \\
\text { (2010) }\end{array}$ \\
\hline Évaluation de la performance & importante & faible & $\begin{array}{l}\text { Maignan et al. (2002) ; } \\
\text { Cousins et Spekman } \\
\text { (2003) ; Gimenez et Sierra } \\
\text { (2013) }\end{array}$ \\
\hline Relationnel & Faible (-) & $\rightarrow$ Élevée $(++)$ & \\
\hline Formalisation de l'échange & élevée & faible & $\begin{array}{l}\text { Theodorakopoulos et al. } \\
\text { (2005) ; Walker et Bram- } \\
\text { mer (2009) ; Leire et Mont } \\
\text { (2010) }\end{array}$ \\
\hline $\begin{array}{l}\text { Partage de } \\
\text { l'information/ressources et } \\
\text { compétences }\end{array}$ & faible & élevé & $\begin{array}{l}\text { Carter et Jennings (2000) ; } \\
\text { Halldorsson et al. (2009); } \\
\text { Park-Poaps et Rees (2010) }\end{array}$ \\
\hline $\begin{array}{l}\text { Confiance inter- } \\
\text { organisationnelle }\end{array}$ & faible & élevée & $\begin{array}{l}\text { Krause et al. (1999); An- } \\
\text { dersen et Skjoett-Larsen } \\
\text { (2009) ; Park-Poaps et } \\
\text { Rees (2010) }\end{array}$ \\
\hline $\begin{array}{c}\text { Relations antérieures } \\
\text { d'affaires }\end{array}$ & $\begin{array}{l}\text { préexistentes ou nou- } \\
\text { velles }\end{array}$ & prééxistentes & $\begin{array}{c}\text { Krause et al. (1999) ; Zsi- } \\
\text { disin et Siferd (2001); } \\
\text { Hoejmose et Adrien-Kirby } \\
\text { (2012) }\end{array}$ \\
\hline
\end{tabular}

En résumé, la revue de littérature sur les antécédents intra et inter-organisationnels permet de déboucher sur une grille d'analyse de l'adoption d'une démarche d'AR selon trois phases : la 
mise en place, le déploiement et le maintien (Figure 1). En nous appuyant sur cette représentation, nous cherchons à approfondir les travaux antérieurs qui n’ont pas opéré le lien entre les antécédents et les phases du processus.

Figure 1 - Grille d'analyse du processus d'adoption des AR

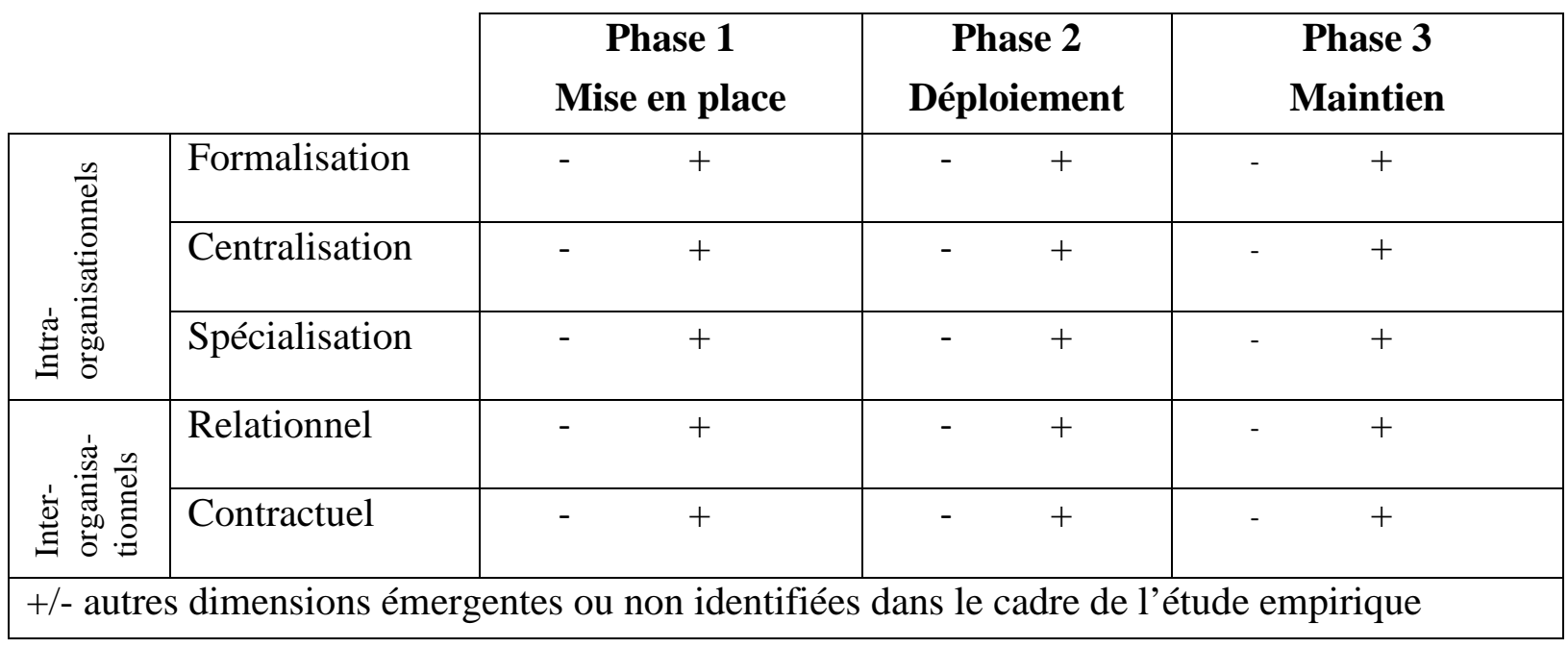

\section{Une étude de cas exemplaire : la SNCF}

Notre propos est de confronter empiriquement notre grille d'analyse (Figure 1), d'identifier la nature des antécédents intra et inter-organisationnels susceptibles d'être présents à chaque phase $^{4}$ et de l'enrichir ou l'amender de dimensions essentielles à la mise en œuvre d'une démarche d'AR. En l'absence de travaux sur les antécédents des AR vus comme un processus, il n’est pas possible de différencier a priori les antécédents selon les phases. Notre démarche, de nature abductive, vise donc à comprendre ce lien ${ }^{5}$. Nous cherchons ainsi à aboutir à des propositions d'explications plutôt qu'à des règles indiscutables. Elle s’appuie sur une méthodologie qualitative d'un cas considéré comme exemplaire et particulièrement avancé dans cette démarche : la SNCF.

\footnotetext{
${ }^{4}$ Cette perspective est conforme à notre positionnement épistémologique qui relève du réalisme critique (Bhaskar, 1975). La réalité, en plus d'exister en soi, est multidimensionnelle, stratifiée, ouverte et différenciée susceptible d'explications diverses, à travers différents mécanismes générateurs (Wynn et Williams, 2012). La combinaison des approches processuelle et organisationnelle (intra et inter) est en phase avec cette vision de la réalité même si les théories mobilisées ne représentent jamais qu’une partie de la réalité, les méthodes utilisées peuvent être faillibles et les capacités cognitives humaines, dont celles des chercheurs eux-mêmes, sont limitées.

${ }^{5}$ Cette recherche vise à décrire et à rendre compte des antécédents instaurés lors de la conduite d'une démarche d'AR. Notre propos est d'enrichir la grille de lecture (Figure 1). Cette démarche d'exploration hybride (Charreire et Durieux, 2003) s'appuie sur une méthodologie qualitative d'une étude de cas approfondie afin de faire émerger les antécédents à l'œuvre en fonction des phases de la démarche.
} 


\subsection{Le choix de la SNCF}

Le recours à l'étude de cas se justifie par la complexité de l'objet avec notamment la vision processuelle du modèle d'adoption. Parce que cette recherche tente de mieux comprendre « comment » mettre en œuvre une démarche d'AR, l'étude de cas est ici retenue. L’étude de cas est une méthode particulièrement justifiée lorsque la question de recherche est large et lorsque l'on souhaite avoir une meilleure compréhension d’un phénomène (Yin, 2013). Le cas SNCF a été choisi pour le potentiel de découverte qu'il représente, mais aussi pour son caractère exemplaire, faisant preuve d'un engagement remarquable en matière d'AR. Cette exemplarité peut être attestée à la fois par l'ancienneté de la démarche et par la légitimité externe de l'entreprise en matière d'AR (Andersen et Skjoett-Larsen, 2009). La SNCF a en effet mis en œuvre la démarche depuis plus de 8 ans, ce qui, en outre, permet une prise de recul des personnes interrogées par rapport à l'objet étudié. En France, cette entreprise est reconnue comme étant la plus mature et active dans le domaine des AR (au regard des nombreux trophées, titres reçus en tant qu'entreprise responsable et de son active participation à l'élaboration de la norme AFNOR sur les AR). Par ailleurs, dans une optique de généralisation des résultats, il est intéressant d'étudier une entreprise intégrant une grande diversité de familles d'achats qui intègre aussi bien des services (service de nettoyage, entretien des voies) que du matériel industriel (matériel roulant) (Krause et al., 1999). Nous n’avons donc pas choisi la SNCF pour son statut d'entreprise publique, mais bien parce qu'il s'agit d'une entreprise exemplaire en matière d'AR. Les rares travaux qui étudient les AR dans les deux contextes (privé vs public) (Walker et Brammer, 2009 ; McMurray et al., 2014) tendent d'ailleurs à conclure à un effet peu significatif ou neutre du caractère public (vs privé) sur les AR ${ }^{6}$.

\subsection{Recueil, validité et traitement des données}

Notre étude de cas unique repose sur un échantillon de dix acteurs distincts (Top managers, middle managers, acheteurs, consultants), avec pour particularité d'avoir tous vécu ou accompagné la démarche d’AR dès son lancement (cf. Tableau 3). Ce choix s’avère essentiel

\footnotetext{
${ }^{6}$ Walker et Brammer (2009) relèvent deux spécificités associées aux organisations publiques en matière d'AR. Elles seraient plus orientées vers les problématiques sociales qu'environnementales et moins ouvertes aux changements que les entreprises privées. Au-delà, les auteurs montrent que les organisations publiques et privées partagent des caractéristiques communes bien plus essentielles telles que la prise en compte de multiples parties prenantes, une concurrence pour l'obtention de ressources et des relations de pouvoir avec leurs fournisseurs et clients. Très récemment, McMurray et $a l$ (2014) étudiant les barrières aux pratiques d'AR indiquent que quel que soit le type d'organisation (publique ou privée), la perception de ces barrières par les responsables achats reste la même.
} 
pour garantir la validité interne de notre étude et nous a permis d’aboutir au phénomène de saturation (Strauss, 1987). Ces entretiens ont été conduits à l'aide d'un guide semi-directif intégrant l'ensemble des dimensions issues de la littérature dont l'architecture est fournie en annexe. L’objectif était de comprendre la manière dont la démarche a été mise en œuvre, d’identifier les différents antécédents intra et inter-organisationnels ayant favorisé cette démarche et leur rôle au cours du processus. Tous les entretiens, de 1 heure 30 en moyenne, ont été enregistrés (soit 15 heures d'entretiens au total). Ils ont fait l'objet d'une retranscription qui correspond à un volume de 262 pages puis d'une validation auprès des interviewés. Par ailleurs, dans un souci de triangulation des données, cette étude a fait également appel à des données secondaires avec le recours à des sources internes (ex. : dispositifs formalisés permettant d'évaluer les acheteurs, supports utilisés lors des Trophées des Achats) et externes (ex : extraits de presse, coupures de journaux, 6ème Baromètre HEC Achats Responsables, étude de cas sur la SNCF de la Darden Business School).

L’analyse des données a été réalisée à partir d’un codage thématique sur les principales catégories héritées de la littérature (centralisation, formalisation, spécialisation, mode relationnel, mode transactionnel, phase d'avancement) et sur une catégorie émergente relative aux antécédents individuels non appréhendés dans la grille d’analyse. La méthode graphique ou dite de mapping a facilité l'étude de la dimension processuelle. Sur la base d'une frise chronologique, chaque répondant était appelé à qualifier les grandes phases de la démarche d'AR à la SNCF, leur nombre et à préciser l'étape atteinte par la SNCF. De même, il était demandé à chaque répondant d'identifier les antécédents intra et inter-organisationnels mobilisés pour chacune des phases mentionnées. 
Tableau 3 - Synthèse des types de données recueillies

\begin{tabular}{|c|l|c|c|}
\hline \multicolumn{1}{|c|}{ Dersonnes interviewées } & \multicolumn{2}{c|}{ Déroulement } \\
\hline \multicolumn{1}{|c|}{ Fonction } & Date & Durée de l'entretien \\
\hline Acteurs & \multicolumn{1}{|c|}{ Pcheteur famille prestations intellectuelles (SNCF) } & $14 / 11 / 2013$ & $01: 55$ \\
CB & Manager stratégies et risques RSE Achats (SNCF) & $07 / 11 / 2013$ & $01: 50$ \\
CW & Consultant Développement Durable (PwC) & $07 / 11 / 2013$ & $01: 43$ \\
DP & Ex-conseiller expert achats responsables (SNCF) & $19 / 11 / 2013$ & $01: 45$ \\
EW & Consultant Achats Responsables (Corel) & $30 / 10 / 2013$ & $01: 40$ \\
FG & Directeur Délégué Achats Durables (SNCF) & $10 / 12 / 2013$ & $01: 20$ \\
OM & Acheteur famille, Direction du Matériel (SNCF) & $29 / 11 / 2013$ & $01: 50$ \\
PD & 2007-2012 : directeur des Achats Groupe (SNCF) & & \\
& Depuis Nov. 2012 : Médiateur des relations inter- & $20 / 12 / 2013$ & $00: 35$ \\
PP & entreprises (Ministère du redressement productif) & $14 / 11 / 2013$ & $01: 40$ \\
RD & Chef de pôle achats Proximités/Fret (SNCF) & $29 / 11 / 2013$ & $01: 20$ \\
VV & Directeur Délégué Services et Opérations (SNCF) & \\
\hline
\end{tabular}

\begin{tabular}{|c|l|}
\hline \multicolumn{2}{|c|}{ Données secondaires } \\
\hline $\begin{array}{c}\text { Données } \\
\text { internes }\end{array}$ & $\begin{array}{l}\text { Indicateurs pour évaluer les acheteurs, support utilisé lors de la soutenance pour les Tro- } \\
\text { phées des Achats }\end{array}$ \\
\cline { 1 - 1 } $\begin{array}{c}\text { Données } \\
\text { externes }\end{array}$ & $\begin{array}{l}\text { Coupures de journaux (les échos, la Tribune), 6ème Baromètre HEC Achats Responsables, } \\
\text { Étude cas sur la SNCF de la Darden Business School }\end{array}$ \\
\hline
\end{tabular}

\section{Des effets différenciés des antécédents selon les trois phases}

Trois résultats clés se dégagent de cette recherche. Tout d'abord, la démarche d'AR est bien structurée autour de trois principales phases. Ensuite, les antécédents tant intraorganisationnels qu’inter-organisationnels diffèrent selon les phases, les antécédents intraorganisationnels précédant les antécédents inter-organisationnels dans la mise en œuvre d'une telle démarche. Enfin, il ressort de l'analyse l’importance d'antécédents non appréhendés au départ, d'ordre individuel, liés aux valeurs et convictions personnelles de certains membres de l’organisation.

\subsection{Les phases clés du processus d'AR}

L’étude révèle que la démarche d’AR peut être découpée en trois principales phases. Si la dénomination diverge selon les acteurs (ex. : ciblage, mise en place et reporting pour le répondant DP ou organisation, structuration et ancrage pour le répondant CW), les phases identifiées correspondent bien aux mêmes périodes au sein de la SNCF. Ainsi, la première phase (2007-2009), que nous pouvons qualifier de mise en place, a débuté en 2007 dans le cadre de la nouvelle politique d'entreprise «En route vers 2012 », sous l'impulsion de deux nouveaux collaborateurs (OM, Directeur Délégué Achats Durables et PP, Directeur des Achats Groupe). Dès sa nomination le Directeur des Achats Groupe a explicitement introduit un axe AR dans le nouveau programme achats appelé « SynergiA » (2008-2012). À cette époque, les compétences détenues en interne sur ce sujet étant limitées, il a souhaité recruter un expert de la thématique pour incarner la fonction de Directeur des AR (OM). Ce dernier a été mandaté 
pour développer cet axe stratégique au sein de la direction des achats. Grâce au soutien de la direction générale, il a pu rapidement recruter, dès 2008, de nouvelles ressources principalement en externe et ainsi créer une équipe dédiée et spécialisée aux AR : la Direction Déléguée aux Achats Durables (DDAD). La nomination du Directeur des AR au CoDir a permis de légitimer sa démarche en interne en faisant du développement durable un axe fort pour la SNCF.

«Mais pour moi ce qui a été fort comme symbole, c'est la direction dédiée, ça paraît bête, mais voilà ça marque et il (OM) siégeait au CoDir. Très vite il y a eu un programme qui s'appelait SynergiA [...] qui de mémoire avait 5 axes dont les Achats Durables. » (DP, Consultant développement durable, 07/11/2013)

Jusqu'en 2009, l'équipe de la DDAD, composée d'une dizaine de collaborateurs, a fait un travail de ciblage, c'est-à-dire un repérage de la nature des enjeux selon les familles : quels enjeux économiques, environnementaux, sociaux, pour quelles familles ? Par exemple, sur la famille «mobilier de gare », la DDAD a identifié que cette famille comprenait des enjeux environnementaux. Elle a donc entrepris un travail de réflexion autour des certifications existantes (PEFC, FSC, etc.). Le plan d'action consistait, dans un premier temps, à informer les acheteurs des enjeux spécifiques et de leur donner des préconisations relatives à leur famille d'achats.

«En fonction de la nature de l'achat, on essaie de voir les enjeux spécifiques de ce secteur d'activité. » (CW, le 07/11/2013)

Ce n’est qu’à partir de fin 2009 que la phase 2 de déploiement en interne a commencé. La DDAD a alors travaillé conjointement avec les responsables stratégie famille (middle management) afin de définir ensemble des critères de développement durable à intégrer directement dans le processus de définition et sélection des offres afin de concrétiser la démarche.

"On les a engagés dans cette dynamique de réflexion en leur donnant comme objectif de sortie une Margueritte à l'image de l'iso 26000 et des 7 questions centrales (en matière de développement durable). Une Marguerite propre à leurs enjeux de département » (CW, Manager Stratégie et Risque RSE Achats, $7 / 11 / 2013)$

Enfin, en phase 3 (2011) de maintien de la démarche, les fournisseurs ont été intégrés et des indicateurs quantitatifs et coercitifs ont été instaurés. L’objectif est de professionnaliser et de crédibiliser la démarche.

"[Concernant les indicateurs de performance des AR] Il y a eu une première phase qui était plutôt light, une deuxième phase où l'on était sur des indicateurs 
mesurés opposables (...) Et depuis, depuis début 2013, donc depuis un an, on a rendu cette mesure objective, on l'a rendue plus coercitive. » (OM, Direction Délégué Achats Durables, 10/12/2013)

\subsection{Les antécédents intra-organisationnels}

La mise en œuvre de la démarche d'AR passe par l'évolution de la structure organisationnelle et la présence d'une équipe de direction dédiée afin d'impulser la dynamique. La communication majoritairement descendante en phase 1 a permis de diffuser largement l'orientation stratégique prise par la direction. L’allocation de moyens dédiés, via une structure Achats Durables chargée de sensibiliser le personnel avec un plan de formations et d'actions, a permis de crédibiliser la démarche en interne. Ainsi durant cette première phase, les acheteurs n’avaient pas d'objectif quantifié, mais ils devaient se sensibiliser aux politiques d'AR via des lectures, visites d'expositions, des formations ludiques en lien avec le développement durable. Notons que cet objectif de sensibilisation a largement été atteint puisque près de $80 \%$ des acheteurs (soit plus de 500) ont également suivi un programme de formation interne sur le sujet. Ce n'est qu'à partir de la phase 2 que des objectifs chiffrés, avec des indicateurs quantitatifs et plus directement en lien avec la fonction achats, sont apparus dans les entretiens annuels individuels. Plus de 85 \% des acheteurs ont aujourd'hui des objectifs de développement durable évalués lors des entretiens individuels avec leur manager. L’implication des managers a été rendue possible grâce au support de la DDAD. La mise en place d'objectifs et de procédures met en exergue un accroissement de la formalisation. Afin de favoriser la communication entre la DDAD et les acheteurs, des Cadès (Coordinateurs Achats Durables et Solidaires) ont été nommés. Il s’agit d'acheteurs, qui sur la base du volontariat, font remonter les objectifs chiffrés à la DDAD et sensibilisent les autres acheteurs de leur équipe à la démarche. Une fois la démarche mise en place, il est nécessaire de l'entretenir afin d'assurer sa pérennité (phase 3).

Durant cette dernière phase, l'objectif est que la DDAD puisse progressivement s'effacer, que chaque acheteur famille et chaque Cadès prennent le relais au sein de son équipe. Toutefois, cette dernière phase reste fragile et n'est pas encore acquise. En effet, les acheteurs opérationnels n'intègrent pas encore complètement la démarche d'AR dans leurs routines. De l'avis des acteurs, il s’agit bien d’un processus et non d'un projet marqué par une date de fin.

"Même si les choses paraissent ancrées, elles restent fragiles. C'est important. Ce qui veut dire qu'il faut garder une vigilance sur cette dimension-là, qui est extrêmement nouvelle et donc extrêmement difficile à ancrer. [...] On peut dire qu'on a quand même réussi à faire pas mal de choses, mais il faut rester assez 
modeste sur le côté ancrage longue durée de ce que l'on a pu réaliser » (PP, exdirecteur des Achats Groupe de la SNCF, 20/12/2013)

\subsection{Les antécédents inter-organisationnels}

L’implication des fournisseurs dans la démarche d'AR ne s'effectue pas en phase amont. Toutefois, si les antécédents intra-organisationnels précèdent et prédominent les antécédents interorganisationnels en phases 1 et 2, ces derniers deviennent ensuite essentiels pour le maintien de la démarche.

«Le fournisseur, c'est vraiment le cour du métier de l'acheteur. On a eu, fin 2010, l'émergence d'un projet qui est devenu une démarche sur le risque et la performance RSE des fournisseurs qui est très structurante pour nous aujourd'hui. C'est vraiment là qu'on a notre belle réussite, ce qui marche le mieux, ce que l'on va poursuivre et ce qui fait basculer aussi les fournisseurs dans la même dynamique, c'est assez puissant cette démarche-là. » (CW, Manager Stratégies et risques RSE Achats, 07/11/2013)

Notons que, dès la phase 2 de déploiement en interne, une réflexion a été menée sur l'évaluation du risque RSE chez les fournisseurs. Ainsi, la DDAD a développé une méthodologie de cartographie du risque au niveau des familles. Lorsqu’une famille présente un risque RSE, une analyse au niveau des fournisseurs est menée (évaluation EcoVadis). Toutefois, ce n'est qu'en phase 3 que certains fournisseurs stratégiques ${ }^{7}$ sont intégrés à la démarche et directement gérés par les acheteurs familles. À partir de leur évaluation EcoVadis (à la charge de la SNCF), les fournisseurs peuvent mesurer leur niveau d'engagement dans cette démarche et voir les aspects sur lesquels ils peuvent développer des marges de progrès. Pour tous ceux dont les résultats sont inférieurs à la moyenne, la DDAD leur demande de mettre en place des plans d'actions correctifs qui sont ensuite pilotés par les acheteurs familles. Une réévaluation à 12 ou 18 mois est alors réalisée. Il s'agit d'un processus d'amélioration continue, où le fournisseur peut être évalué plusieurs fois jusqu’à obtention d’une note supérieure à la moyenne. Ainsi, au cours de la phase 3, les fournisseurs bénéficient d’un accompagnement.

«Ils ont un outil d'évaluation qui s'appelle EcoVadis qui regarde la performance RSE des fournisseurs sur la base d'une note sur 10 [...] Lorsque le fournisseur a une mauvaise note, ils ne vont pas tout de suite le sanctionner et l'enlever du panel. Ils vont le rencontrer [...] On n'est pas dans quelque chose

\footnotetext{
7 Seuls 2 à 3000 fournisseurs dits stratégiques, ou à risques (ex : familles ferroviaires où il y a des enjeux forts de sécurité) sont déjà impliqués dans une démarche RSE depuis plusieurs années. Leur performance est suivie par une centaine de qualiticiens (issus du service qualité de la SNCF (DDQF) à l'aide d'un système d'amélioration continue basée sur 7 axes standards (dont un axe dédié à la RSE). Cette évaluation est réalisée de manière documentaire ou sur le terrain.
} 
d'autoritaire [...] Ils essaient de comprendre et d'améliorer » (DP, Consultant développement durable, 7/11/2013)

La démarche collaborative en externe semble beaucoup moins aboutie qu'en interne, touchant essentiellement aux fournisseurs stratégiques. Le verbatim ci-dessus montre qu'elle est davantage basée sur un mode relationnel.

\subsection{Les antécédents individuels}

Outre les antécédents intra et inter-organisationnels, les entretiens confirment l'importance des caractéristiques individuelles des parties prenantes à la démarche, telles les valeurs et la personnalité des managers qui impulsent la démarche.

La sensibilité personnelle et la conviction d'agir dans le bon sens poussent certains acteurs à porter la démarche, quitte à bousculer certaines personnes réticentes. L'ensemble des personnes interrogées est unanime pour souligner le rôle clé de deux protagonistes : PP (exDirecteur des Achats Groupe) et OM (Directeur Délégué Achats Durables).

«C'est sûr, au début, il y avait PP (...), il avait une forte sensibilité au développement durable; et en fait, c'est lui qui a recruté OM, (...). Il voulait avoir un axe développement durable à part entière. Pour lui, c'était une volonté forte que d'avoir un axe achats durables au niveau de sa direction achats » (EW, exconseiller expert achats responsables, le 19/11/2013)

«OM fut porteur de cette démarche au sein de la direction des achats, avec beaucoup de conviction, beaucoup d'implication. Oui, il y croit. Il y croit vraiment, on sent que c'est ancré en lui, c'est dans son $A D N$, il sait bien en parler, il sait bien faire adhérer. » (VV, Directeur Délégué Services et Opérations, le 29/11/2013)

Plusieurs éléments montrent que PP et OM sont guidés par une conviction profonde et non par de l'opportunisme professionnel. Ainsi, ils participent activement, et ce même avant d'avoir rejoint la SNCF, à des groupes de discussion et à des associations en lien avec la RSE et les Achats. PP a notamment été président de l'association CDAF (Compagnie des Dirigeants et Acheteurs de France) et de pas@pas dont le but est de favoriser l'insertion professionnelle des personnes handicapées. Il a également participé à la création du Pacte PME, de la charte des relations fournisseurs responsables et de l'ObSAR. Concernant OM, c'est après avoir exercé comme acheteur qu'il a pris conscience qu'il ne pouvait plus ignorer l'impact négatif que pouvait avoir cette fonction achats.

«Il y a 10 ans ou 20 ans, j'ai quitté la fonction achats en claquant la porte. J'étais chez $X$ à l'époque et je me disais : c'est une fonction de tarés, ils ne pen- 
sent qu'au pognon, ils ne pensent qu'à la réduction des prix, sans regarder les impacts que ça peut avoir sur les fournisseurs, sur les écosystèmes. Moi, j’allais acheter toujours plus loin, dans les pays low cost (...). Un jour, je suis tombé de ma chaise et je me suis dit : je ne suis quand même pas né pour détruire de la valeur... (OM, Directeur Délégué Achats Durables, 10/12/2013)

$\mathrm{OM}$ et $\mathrm{PP}$ ont, par ailleurs, une vision à long terme, leur ambition de faire passer les AR dans les routines achats dépasse largement le cadre de la SNCF. Ils cherchent à convaincre d'autres organisations de la pertinence de la démarche. À noter que PP était au moment des interviews médiateur des relations inter-entreprises auprès du ministre du redressement productif.

Les résultats montrent que ces individus convaincus n’appartiennent pas uniquement à la hiérarchie, et c'est en prenant conscience de ceci que la SNCF a su construire une condition du succès du déploiement de sa démarche. Elle a identifié les «bons » porte-parole et a pu ainsi constituer un réseau de convaincus en interne : les Cadès. Nommés par leur chef de département, les candidats au poste de «Cadès » ne bénéficient pas de rémunération pour cette mission complémentaire. Ils sont volontaires et sont motivés intrinsèquement, par la nature d’une mission dite «stimulante et enrichissante », par une animation du club Cadès jugée « sympathique » et par une reconnaissance interne de leur travail.

«À la base, c'est vrai qu'il faut une conviction personnelle de quelqu'un qui soit suffisamment élevé dans le niveau de management (...) ; et puis, un minimum d'équipe et de connaissance pour pouvoir porter le message. » (PP, exdirecteur des Achats Groupe, le 20/12/2013)

"Au niveau de mon pôle, j'ai une Cadès et elle prend ça très, très à cœur. Elle est dans tous les dossiers, c'est-à-dire que les acheteurs se tournent vers elle, pour avoir des informations, ou bien pour savoir où trouver la bonne réponse, ou savoir vers qui s'orienter. Elle est très sollicitée dans le département et puis elle prend son rôle très à cœur, donc oui, c'est très bien. » (RD, Chef de pôle achats Proximités/Fret, le 14/11/2013)

La figure 2 fournit une synthèse des résultats. Elle montre que les antécédents intra et interorganisationnels ne sont pas statiques et qu'ils jouent un rôle différencié selon les trois phases. La démarche est d’abord structurée en interne avant d’être étendue aux fournisseurs. La grille d'analyse proposée à l'issue de la revue de littérature a ainsi été enrichie et propose désormais une analyse fine de la nature des antécédents au sein de chaque phase du processus. Des antécédents individuels ont également été introduits, car essentiels dans les phases amont. Les valeurs et la conviction de certains dirigeants apparaissent comme essentielles pour impulser la démarche lors de la phase de mise en place. Ces mêmes valeurs et convictions doivent ensuite être relayées par le middle management puis par les salariés afin d'assurer le déploiement de la démarche en interne. 
Trois propositions principales (P1, P2 et P3) peuvent être formulées à partir de la figure 2 qui synthétise nos résultats. Celles-ci pourront faire l’objet d'une validation lors de futures recherches.

P1 : La probabilité de mettre en œuvre une démarche d'AR sera d'autant plus forte que :

- les membres de la direction sont convaincus et engagés dans la démarche,

- la centralisation est élevée et la formalisation limitée,

- les fournisseurs ne sont pas intégrés à la démarche.

P2 : La probabilité de déployer une démarche d’AR sera d'autant plus forte que :

- l'implication et le soutien de la direction perdurent,

- la décentralisation et la diffusion des responsabilités s'accompagnent d'une formalisation via la standardisation des procédures,

- le risque RSE des fournisseurs est évalué.

P3 : La probabilité de pérenniser une démarche d'AR sera d’autant plus forte que :

- l'ensemble des salariés est engagé dans la démarche,

- le management est davantage participatif avec une dissémination des savoirs à toute l’organisation et un degré de formalisation toujours important,

- les fournisseurs sont incités contractuellement et accompagnés afin de s’engager dans la démarche d'AR du donneur d'ordre. 


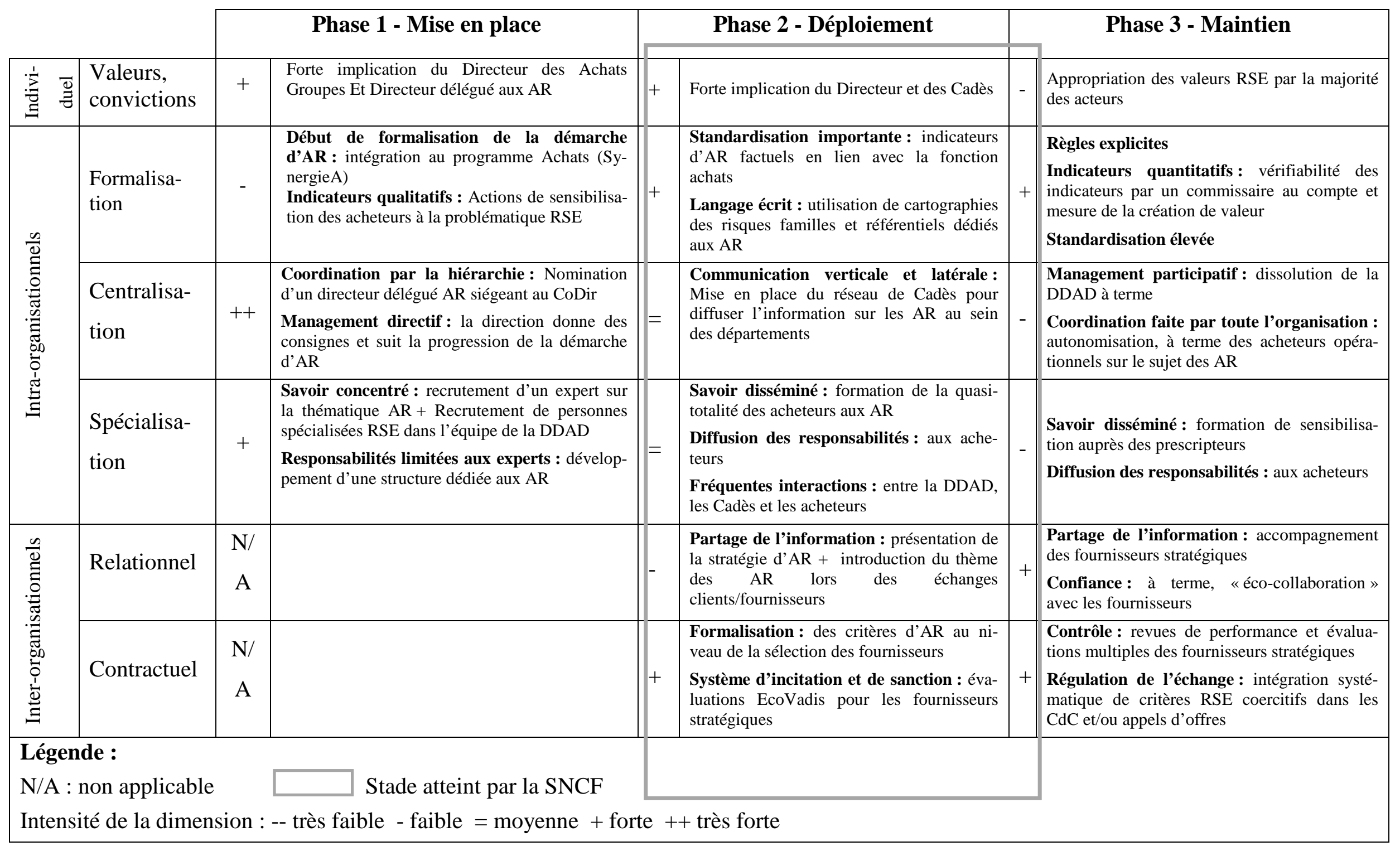




\section{Discussion et conclusion}

Le cas SNCF corrobore les conclusions de Bowen et al. (2001) et Igarashi et al. (2013), selon lesquelles les leviers des AR sont plutôt proactifs que réactifs et qu'une chaîne d'approvisionnement « verte » passe nécessairement par une stratégie proactive. Le cas SNCF conforte aussi l'approche stratégique de la RSE qui associe aux comportements proactifs (versus réactifs) une performance supérieure, notamment à long terme (Sharma et Vredenburg, 1998 ; Porter et Kramer, 2006). À la différence de la grande majorité des travaux sur les antécédents sur les AR, notre recherche précise aussi que ce comportement plutôt proactif doit être observé tout au long du processus d'adoption, et pas seulement au moment de la décision. Elle montre que ce comportement plutôt proactif est essentiellement porté par la direction dans les phases amont. En revanche, avec l'avancée de la démarche l’intégration progressive des parties prenantes semble garantir la pérennité de la démarche. Au niveau interorganisationnel, nos résultats sont dans la même veine que Lavastre et al. (2014) qui étudient les pratiques inter-organisationnelles innovantes et montrent l'importance de manager les fournisseurs afin qu'ils soient en capacité d'intégrer ce processus. Le cas SNCF permet de préciser ce résultat en montrant l’influence retardée des pratiques inter-organisationnelles. Ils soulignent l'importance des leviers intra et inter-organisationnels, tout en établissant leur caractère séquentiel.

Dans la lignée des travaux de Porter et Kramer (2006), nos résultats rendent compte de l'importance de l'alignement entre la stratégie d'AR de la SNCF et les dispositifs intra et inter-organisationnels nécessaires à sa pérennité. Bien qu’importante, cette volonté d'instaurer une démarche d'AR ne suffit pas à elle seule pour obtenir les résultats escomptés. Encore faut-il que cette stratégie soit en adéquation avec la stratégie d’affaires (Business) de l'entreprise. C’est ce phénomène qui est visé par le concept d'alignement stratégique. Pour que les décisions prises au niveau du top management ne deviennent pas des intentions stériles, une vigilance particulière doit être portée au déploiement de cette démarche d'AR à tous les niveaux de l’organisation. Ainsi, une structure centralisée et une spécialisation des compétences autour d’une équipe dédiée semble préférable lors de la mise en place de la démarche. Ceci vient illustrer le modèle d'alignement stratégique de Cousins et Spekman (2003) qui souligne l'importance d'aligner la stratégie avec le niveau de compétence et de qualification des acheteurs et le système de mesure de la performance. Le cas SNCF vient étayer cette idée puisque de manière séquentielle, les acheteurs ont été formés, les objectifs opérationnels redé- 
finis, les processus liés aux métiers d'acheteurs mis en conformité (validation au panel, formulation des appels d’offres, dépouillement des offres, etc.), et les systèmes de mesure de la performance réajustés en fonction de ce nouvel axe stratégique. La centralisation et la spécialisation de la démarche permettent de la rendre visible et crédible. Sur ce point, nos résultats sont en lien avec ceux de Calvi (2000) sur la nécessité de développer des postes spécifiques. La phase de déploiement repose, quant à elle, sur une formalisation de la démarche au travers d’objectifs chiffrés et de codes de conduite. Cette formalisation est couplée à une décentralisation via l'implication des Cadès et du middle management. Ces derniers jouent un rôle clé de diffusion et d'appropriation progressive de la démarche par les acheteurs familles. Enfin, la phase de maintien, qui n'est pas encore complètement acquise à ce jour, repose, en interne, sur une décentralisation plus poussée, l'étape ultime étant marquée par la disparition de la structure dédiée aux AR.

Concernant les antécédents inter-organisationnels, ils ne semblent intervenir qu’à une phase déjà avancée de la démarche. Si la dimension contractuelle apparaît comme essentielle en phase de déploiement, elle devient étroitement liée à la dimension relationnelle en dernière phase. Nous rejoignons Lim et Phillips (2008) qui montrent que les codes de conduites et audits fournisseurs (mécanismes propres à un mode de gouvernance contractuel) ne sont pas suffisants pour mener une politique RSE. Ceci conforte l'idée selon laquelle ces deux dimensions sont davantage complémentaires que substituables (Andersen et Skjoett-Larsen, 2009). Les résultats suggèrent ainsi que l'aspect coopératif n’émerge qu'après avoir atteint un niveau suffisant de maturité relationnelle. Comme le soulignent les travaux adoptant une approche d'essence sociologique, le développement de relations inter-organisationnelles nécessite la préexistence de relations entre les organisations et, surtout, entre les individus qui composent ces organisations. Le courant sociologique vise à offrir une représentation plus convaincante de la réalité des organisations. Il reproche au courant économique la non prise en compte des relations sociales dans lesquelles tout acteur économique réel est inscrit (Burt, 1982 ; Reynaud, 1988). Les interactions développent des transactions humainement satisfaisantes plutôt qu'économiquement optimales. Les résultats de cette recherche montrent également que les relations inter-organisationnelles ne peuvent pas être uniquement appréhendées par les rapports économiques entre les individus et les groupes le constituant. En effet, les règles communes d'échange (normes, valeurs, etc.) instaurent des barrières à l'entrée et à la sortie permettant aux fournisseurs stratégiques de se protéger des risques d'opportunisme et de relations court-termistes. De plus, l'existence de relations de confiance facilite la rapidité 
d'exécution et la flexibilité nécessaire à la mise en place d'une démarche d'AR. Toutefois, comme le soulignent Andersen et Skjoett-Larsen (2009), cette confiance n’exclut pas le recours à des dimensions contractuelles. Ainsi, l’utilisation de mécanismes contractuels (contrats, sanction, incitation) renforce les chances de voir la confiance émerger entre les fournisseurs et le donneur d'ordre, car les risques et l'incertitude de la coopération sont partiellement maîtrisés. Les mécanismes contractuels fournissent alors un guide des comportements à adopter pour que les relations inter-organisationnelles soient performantes (Das et Teng, 1998).

Par ailleurs, cette recherche nous conduit à enrichir notre revue de la littérature sur un aspect non appréhendé au départ. Les résultats révèlent à travers la nomination d'un Directeur Délégué aux AR et la présence des Cadès que la démarche d'AR est difficile à instaurer sans des valeurs et des convictions individuelles (Drumwright, 1994 ; Carter et Jennings, 2004). Elles sont motrices dans la mise en œuvre d'une démarche d'AR. Ainsi, les acteurs du changement (Carassus, et al., 2014), parfois appelés « policy entrepreneurs » (Drumwright, 1994) ou «value champion » (Walker et al., 2008) donnent un élan au processus d’AR. Drumwright (1994) les décrit comme des personnes disposées à investir leur temps, leur énergie, leur argent et leur réputation dans l'espoir d'un changement organisationnel qui soit en lien avec leur valeur. Quelle que soit leur position hiérarchique, ces individus ont une force de persuasion qui leur permet de convaincre de l’intérêt de la démarche et d'accroître l'adhésion et la participation des équipes (Caniëls et al., 2013). Ces individus, souvent passionnés, sont plus efficaces lorsque les décisions ne sont pas centralisées, c’est-à-dire lorsque le top management n'impose pas les règles de conduite. Un fort degré d'autonomie des individus s'avère favorable à la mise en œuvre d'une démarche d'AR (Drumwright 1994). Ainsi, il est souhaitable de laisser libre d'agir indépendamment les policy-entrepreneurs, tout en leur apportant un support hiérarchique. Bien que les thématiques ne soient pas les mêmes, cette étude rejoint en partie les conclusions de l'étude Carassus et al. (2014) sur le rôle clé des « agents du changement ». Elle permet aussi de nuancer le caractère linéaire du processus en introduisant la réflexivité des acteurs et leur capacité à influencer la démarche d'AR. En particulier, cette recherche montre comment ces acteurs du changement peuvent faire tomber certaines barrières aux AR, telles que le cynisme ou l'inertie, identifiées par Harwood et Humby (2008). Le cynisme relève d’individus cherchant à décrédibiliser la démarche (Drumwright, 1994). La direction déléguée aux AR de la SNCF a également été confrontée à « pas mal de gens qui ont été des détracteurs, de grands directeurs en interne »(OM, Direction Délégué Achats Durables, 10/12/2013) que l'on pourrait qualifier de cyniques, ces derniers jugeant la fonction 
AR illégitime. Quant à l'inertie des membres de l'organisation, elle est à mettre en lien avec un système de mesure de la performance trop souvent orienté sur les performances économiques et ignorant les critères non économiques (Harwood et Humby, 2008). Notre étude va plus loin en montrant que la mise en place d'indicateurs d'AR, si elle s'avère nécessaire n'est pas suffisante pour dépasser l’inertie susceptible d'être présente à chaque phase.

Au total, cette recherche est, à notre connaissance, la première à proposer une vision intégrative des antécédents des AR, la grande majorité des recherches ayant porté l'accent soit sur les leviers intra-organisationnels, soit sur les leviers inter-organisationnels (Drumwright, 1994 ; Worthington et al., 2008 ; Worthington, 2009). Nous ajoutons à ces leviers organisationnels, ceux relatifs au niveau individuel. En mobilisant une approche processuelle des AR, elle contribue à une littérature encore émergente dans la lignée de Theodorakopoulos et al. (2005). Elle permet ainsi de mieux comprendre la manière dont les AR se développent et évoluent, tant au niveau des antécédents individuels, organisationnels qu'inter-organisationnelles, là où les réponses théoriques restent partielles. Par ailleurs, elle conduit à enrichir les travaux sur l'adoption d'une telle démarche qui peinent à intégrer explicitement les relations clientfournisseur alors qu’ils semblent jouer un rôle clé sur sa pérennité.

D’un point de vue managérial, cette recherche permet d'identifier les leviers actionnables par les organisations privées et publiques désireuses de s’engager dans une démarche d'AR ou soucieuses de pérenniser leur démarche. La grille d'analyse développée apporte des clés pour créer les conditions propices contribuant au succès d’une telle démarche. Il s'avère être un complément utile à la norme NF X 50-135 qui, dans sa version actuelle, ne prend pas en compte le stade d'avancement dans la démarche d'AR. Il invite aussi les dirigeants à une certaine prudence vis-à-vis des modèles de maturité des AR (Bruel et al., 2009) qui débouchent sur une liste de bonnes pratiques sans préciser les conditions de leur mise en œuvre. De ce point de vue, cette recherche apporte une première brique en réponse au déficit évoqué par Pohl et Förstl (2011) relatif au manque d’outils managériaux adaptés pour mettre en place de manière efficiente une politique d'AR. À l'issue de cette recherche, quatre recommandations à destination des managers peuvent être formulées : 1) Réaliser un diagnostic à partir d'une matrice « antécédents * phases du processus » afin d’identifier les leviers actionnables selon la phase d'avancement atteinte par l'entreprise ; 2) Repérer et mobiliser les porte-parole susceptibles de jouer le rôle de policy entrepreneur ; 3) Construire les conditions organisationnelles propices à l'avancement de la démarche d'AR en interne avant d'impliquer les fournis- 
seurs ; 4) Développer les relations collaboratives avec les fournisseurs pour garantir la pérennité de la démarche à long terme.

Cette recherche n’est pas exempte de limites et des travaux ultérieurs permettraient de les dépasser. D’une part, les approches organisationnelle et processuelle d'essence mécaniste et linéaire que nous avons mobilisées conduisent à sous-estimer les stratégies individuelles des acteurs aux intérêts parfois contradictoires (Crozier et Friedberg, 1977) et la manière dont ils développent leurs actions pour produire les règles, les normes ou les conventions (Reynaud, 1988). Or, les interactions entre les individus, leurs rapports de force sont susceptibles de provoquer des incidents, des tensions entre des priorités (Harwood et Humby, 2008). Des travaux dans le champ de l'innovation montrent que les processus d'adoption de nouvelles pratiques organisationnelles sont complexes, collectifs, systémiques et longs et peuvent intégrer différentes dimensions politiques, cognitives et interprétatives (Damanpour et Aravind, 2012). Cette perspective constitue une voie intéressante pour approfondir l'étude du processus d'AR dans une approche plus itérative ${ }^{8}$. Elle permettrait d'enrichir la lecture adoptée quelque peu schématique pour saisir la réalité étudiée dans toute sa complexité. D’autre part, l'étude révèle que les AR ne reposent pas seulement sur la capacité du fournisseur à adopter des codes de bonnes conduites, mais sur le développement de relations collaboratives client-fournisseur. Dans ce cadre, une question posée est celle du développement d'une base commune de connaissances, des capacités d'apprentissage à long terme (Teece, 1998) et des mécanismes par lesquels une telle capacité peut être développée dans la chaîne d'approvisionnement (Dyer et Nobeoka, 2000). L’étude des antécédents inter-organisationnels pourrait être affinée en intégrant également la perception des fournisseurs dans la mise en œuvre de cette démarche. Par ailleurs, dans une perspective de généralisation des résultats et de test de nos propositions, des études menées sur de larges échantillons d'entreprises pourraient être conduites. Enfin, la prise en compte d'autres facteurs de contingence permettrait de cerner d'éventuelles spécificités liées notamment aux caractéristiques individuelles des entreprises (dont leur statut privé vs public), mais aussi à leur environnement concurrentiel et réglementaire.

\footnotetext{
8 Nous remercions l'un de nos rapporteurs pour cette piste de recherche.
} 


\section{Références}

Andersen M. et Skjoett-Larsen T. (2009), «Corporate Social Responsibility in Global Supply Chains », Supply Chain Management : An International Journal, vol. 14, n 2, p. 5-86.

Bhaskar R. (1975), A Realist Theory of Science, Harvester Press.

Bessant J., Raphael K. et Kaplinsky R. (2003), « Putting Supply Chain Learning into Practice », International Journal of Operations \& Production Management, vol. 23, n 2, p. 67-184.

Blome C. et Paulraj A. (2013), « Ethical Climate and Purchasing Social Responsibility: A Benevolence Focus », Journal of Business Ethics, vol. 116, n 3, p. 567-585.

Bowen F.E., Cousins P.D., Lamming R.C. et Farukt A.C. (2001), « The Role of Supply Management Capabilities in Green Supply », Production and Operations Management, vol. 10, $\mathrm{n}^{\circ} 2$, p. 74-189.

Burt R.S. (1992), Structural Holes, Harvard University Press.

Bruel O., Menuet O. et Thaler P.F. (2009), Livre blanc - Les Achats Durables : un levier essentiel de la sortie de crise, Ecovadis.

Burns T.E. et Stalker G.M. (1961), The Management of Innovation, Tavistock.

Calvi R. (2000), « Le rôle des services achats dans le développement des produits nouveaux : une approche organisationnelle », Finance Contrôle Stratégie, vol. 3, n 2, p. 1-55.

Caniëls M.C.J., Gehrsitz M.H. et Semeijn J. (2013), «Participation of Suppliers in Greening Supply Chains: an Empirical Analysis of German Automotive Suppliers », Journal of Purchasing and Supply Management, vol. 19, $\mathrm{n}^{\circ}$ 3, p. 134-143.

Carassus D., Favoreu C. et Gardey D. (2014), « Les déterminants de l'adoption et de la mise en œuvre d'un management par la performance : application aux collectivités locales françaises », Finance Contrôle Stratégie, vol. 17, n 1, p. 1-24.

Carter C.R. et Carter J.R. (1998), «Interorganizational Determinants of Environmental Purchasing: Initial Evidence from the Consumer Products Industries », Decision Sciences, vol. 29, $n^{\circ} 3$, p. 659-684.

Carter C.R. et Jennings M. (2000), Purchasing's Contribution to the Socially Responsible Management of the Supply Chain, Center for Advanced Purchasing Studies, Tempe, AZ.

Carter C.R. et Jennings M. (2002), "Logistics Social Responsibility: an Integrative Framework », Journal of business logistics, vol. 23, $\mathrm{n}^{\circ} 1$, p. 45-180.

Carter C.R. et Jennings M. (2004), « The Role of Purchasing in Corporate Social Responsibility: a Structural Equation Analysis », Journal of Business Logistics, vol. 25, n² 1, p.145-186.

Charreire S. et Durieux F. (2003), "Explorer et Tester : deux voies pour la Recherche » in R.A. Thietart, Méthodes de Recherche en Management, Dunod.

Closs D.J., Speier C. et Meacham N. (2011), "Sustainability to Support End-to-End Value Chains: the Role of Supply Chain Management », Journal of the Academy of Marketing Science, vol. 39, $\mathrm{n}^{\circ}$ 1, p. 101-116.

Cooper R.B. et Zmud R.W. (1990), «Information Technology Implementation Research : a Technological Diffusion Approach », Management Science, vol. 36, n² 2, p. 123-139.

Cousins P.D. et Spekman R. (2003), " Strategic Supply and the Management of Inter-and IntraOrganisational Relationships », Journal of Purchasing and Supply Management, vol. 9, $\mathrm{n}^{\circ} 1$, p. 19-29.

Crozier M. et Friedberg E. (1977), L'acteur et le système. Les contraintes de l'action collective, éd. du Seuil : Collection « Points Essais».

Damanpour F. et Aravind D. (2012), « Managerial Innovation: Conceptions, Processes, and Antecedents ", Management and Organization Review, vol. 8, n² 2, p. 423-454

Das T.K. et Teng B.S. (1998), «Between Trust and Control: Developing Confidence in Partner Cooperation in Alliances », Academy of management review, vol. 23, n 3, p. 491-512. 
Drumwright M.E. (1994), « Socially Responsible Organizational Buying: Environmental Concern as a Noneconomic Buying Criterion ", Journal of Marketing, vol. 58, n 3, p. 1-19.

Dyer J. et Nobeoka K. (2000), «Creating and Managing a High Performance Knowledge-Sharing Network: the Toyota Case », Strategic Management Journal, vol. 21, p. 345-367.

Emmelhainz M.A. et Adams R.J. (1999), «The Apparel Industry Response to "Sweatshop » Concerns : A Review and Analysis of Codes of Conduct », Journal of Supply Chain Management, $\mathrm{n}^{\circ} 35$, vol. 2, p. 51-57.

Gimenez C. et Sierra V. (2013), «Sustainable Supply Chains: Governance Mechanisms to Greening Suppliers », Journal of Business Ethics, vol. 116, n 1, p. 189-203.

Halldorsson A., Kotzab H. et Skjott-Larsen T. (2009), "Supply Chain Management on the Crossroad to Sustainability: a Blessing or a Curse? », Logistics. Research, vol. $1, \mathrm{n}^{\circ} 2$, p. 83-94

Harwood I. et Humby S. (2008), « Embedding Corporate Responsibility into Supply: A Snapshot of progress », European Management Journal, vol. 26, n 3, p. 166-174.

Hoejmose S.U. et Adrien-Kirby A.J. (2012), « Socially and Environmentally Responsible Procurement: A Literature Review and Future Research Agenda of a Managerial Issue in the 21st Century », Journal of Purchasing and Supply Management, vol. 18, n 4, p. 232-242.

Hult G.T.M., Hurley R.F., Giunipero L.C. et Nichols E.L. (2000), « Organizational Learning in Global Purchasing: A Model and Test of Internal Users and Corporate Buyers », Decision Sciences, $n^{\circ}$ 31, vol. 2, p. 293-325.

Igarashi M., De Boer L. et Fet A.M. (2013), «What is Required for Greener Supplier Selection ? A Literature Review and Conceptual Model Development », Journal of Purchasing and Supply Management, vol. 19, $\mathrm{n}^{\circ}$ 4, p. 247-263.

Krause D.R., Ragatz G.L. et Hughley S. (1999), "Supplier Development from the Minority Supplier's Perspective », Journal of Supply Chain Management, vol. 35, n 3, p. 33-41.

Lavastre O. Ageron B. et Chaze-Magnan L. (2014), « La performance des pratiques interorganisationnelles innovantes », Revue Française de Gestion, vol. 2, n² 239, p. 75-89.

Leire C. et Mont O. (2010), "The Implementation of Socially Responsible Purchasing ", Corporate Social Responsibility and Environmental Management, vol. 17, n 1, p. 27-39.

Lim S.J. et Phillips J. (2008), « Embedding CSR Values: The Global Footwear Industry's evolving Governance Structure », Journal of Business Ethics, vol. 81, n 1, p. 143-156.

Maignan I., Hillebrand B et Mcalister D. (2002), « Managing Socially-Responsible Buying: How to Integrate Non-Economic Criteria into the Purchasing Process », European Management Journal, vol. 20, n 6, p. 641-648.

McMurray A.J., Islam M.M., Siwar C. et Fien J. (2014), « Sustainable Procurement in Malaysian Organizations: Practices, Barriers and Opportunities », Journal of Purchasing and Supply Management, vol. 3, n 20, p. 195-207.

Mont O. et Leire C. (2009), «Socially Responsible Purchasing in Supply Chains: Drivers and Barriers in Sweden », Social Responsibility Journal, vol. 5, n 3, p. 388-407.

ObsAR (2013), Baromètre des Achats Responsables 2013.

Park-Poaps H. et Rees K. (2010), "Stakeholder Forces of Socially Responsible Supply Chain Management Orientation », Journal of Business Ethics, vol. 92, p. 305-322

Pohl M. et Förstl K. (2011), «Achieving Purchasing Competence through Purchasing Performance Measurement System Design, A Multiple-Case Study Analysis », Journal of Purchasing and Supply Management, vol. 17, n 4, p. 231-245.

Poppo L. et Zenger T. (2002), « Do Formal Contracts and Relational Governance Function as Substitutes or Complements? », Strategic Management Journal, vol. 23, p. 707-725.

Porter M. et Kramer M.R. (2006), «Strategy and Society: The Link Between Competitive Advantage and Corporate Social Responsibility », Harvard Business Review, vol. 84, $\mathrm{n}^{\circ} 12$, p. 78-92. 
Reynaud J.D. (1988), « Les régulations dans les organisations : régulation de contrôle et régulation autonome », Revue Française de Sociologie, vol. 29, n 1, p. 5-18.

Sarkis J., Zhu Q. et Lai K. (2011), « An Organizational Theoretic Review of Green Supply Chain Management Literature », International Journal of Production Economics, n 130, p. 1-15.

Sharma S. et Vredenburg H. (1998), "Proactive Corporate Environmental Strategy and the Development of Competitively Valuable Organizational Capabilities », Strategic Management Journal, vol. 19, $\mathrm{n}^{\circ}$ 8, p. 729-753.

Sine W.D., Mitsuhashi H. et Kirsch D.A. (2006), « Revisiting Burns and Stalker: Formal Structure and New Venture Performance in Emerging Economic Sectors », Academy of Management Journal, vol. 49, n 1, p. 121-132.

Strauss A.L. (1987), Qualitative Analysis for Social Scientists, Cambridge University Press.

Tate W.L., Ellram L.M. et Dooley K.J. (2012), « Environmental Purchasing and Supplier Management (EPSM) », Journal of Purchasing and Supply Management, vol. 18, n 3, p. 173188

Teece D.J. (1998), « Capturing Value from Knowledge Assets: the New Economy, Markets for Know-How and Intangible Assets », California Management Review, vol. 40, n 3, p. 55 79.

Theodorakopoulos N., Ram M. et Shah M. (2005), « Experimenting with Supply Chain Learning (SCL): Supplier Diversity and Ethnic Minority Businesses », The International Entrepreneurship and Management Journal, vol. 1, n 4, p. 461-478.

Vachon S. et Klassen R.D. (2008), «Environmental Management and Manufacturing Performance: The Role of Collaboration in the Supply Chain ", International Journal of Production Economics, Vol. 111, n² 2, p. 299-315

Walker H. et Brammer S. (2009), «Sustainable Procurement in the United Kingdom Public Sector », Supply Chain Management : An International Journal, vol. 14, n 2, p. 28-137.

Walker H., Di Sisto L. et Mcbain D. (2008), « Drivers and Barriers to Environmental Supply Chain Management Practices: Lessons from the Public and Private Sectors », Journal of purchasing and supply management, vol. 14, $\mathrm{n}^{\circ} 1$, p. 69-85.

Worthington I. (2009), « Corporate Perceptions of the Business Case for Supplier Diversity: How Socially Responsible Purchasing Can Pay », Journal of Business Ethics, vol. 90, n 1, p. 47-60.

Worthington I., Ram M. et Boyal H. (2008), « Researching the Drivers of Socially Purchasing: a Cross-National Study of Supplier Diversity Initiatives », Journal of Business Ethics, vol. 79, n 3, p. 319-331.

Wynn J.D. et Williams C.K. (2012), «Principles for conducting Critical Realist Case Study Research in Information Systems ", Management Information Systems Quarterly, vol. 36, $\mathrm{n}^{\circ}$ 3, p. 787-810.

Yin R.K. (2013), Case study research : Design and methods, Sage publications.

Zsidisin G.A. et Siferd S.P. (2001), «Environmental Purchasing: a Framework for Theory Development », European Journal of Purchasing \& Supply Management, vol. 7, $\mathrm{n}^{\circ} 1$, p. 61-73. 
Annexe: Arborescence du guide d'entretien

Thème 1. La stratégie d'AR et sa déclinaison

- $\quad$ Sous-thème 1.1. Démarches RSE et AR au sein de l'entreprise

- Sous-thème 1.2. Histoire et phases de la démarche

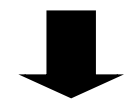

Thème 2. L'organisation interne

Identification des antécédents intra-organisationnels de la démarche d'AR

- Sous-thème 2.1. Spécialisation

- Sous-thème 2.2. Relations horizontales vs verticales

- Sous-thème 2.3. Formalisation/informatisation,

- $\quad$ Sous-thème 2.4. Centralisation/décentralisation.

- $\quad$ + Point de viıe rétrnsnertif

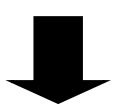

Thème 3. Les relations inter-organisationnelles Identification des antécédents inter-organisationnels de la démarche d'AR :

- $\quad$ Sous-thème 3.1. Mode contractuel

- $\quad$ Sous-thème 3.2. Mode relationnel

- $\quad$ + Point de vue rétrospectif

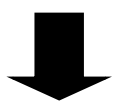

Thème 4. L'influence d'autres antécédents qui n’auraient pas été pris en compte

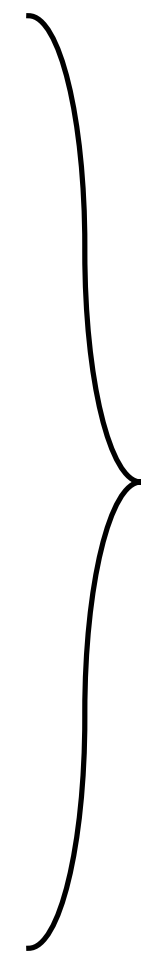

Point de vue rétrospectif : A l'aide d'une frise chronologique, repérage des antécédents intra et inter-organisationnels selon les différentes phases du processus identifiées dans le thème 1.

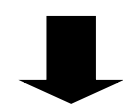

\section{Conclusion}

- Impacts de la démarche sur l'ensemble des parties prenantes (internes et externes)

- Autres sujets importants relatifs aux AR 